\title{
空间激光通信技术发展现状及展望
}

\author{
王天枢，林鹏，董芳，刘显著，马万卓，付强 \\ (长春理工大学空间光电技术国家地方联合工程研究中心, 长春 130022)
}

\begin{abstract}
摘要：空间激光通信技术是未来空间宽带信息传输的主要通信技术，具有带宽高、传输快速便捷及成本低的优势，是解决信 息传输 “最后一千米” 的最佳选择。本文旨在系统把握空间激光通信技术的发展脉络，系统梳理了国内外空间激光通信技术 在星地、星间、空地、空空等链路的研究与试验验证的发展情况, 总结了激光通信技术在捕获跟踪、通信收发、大气补偿和 光机设计等方向的关键技术研究热点。在此基础上，面向未来需求，归纳了空间激光通信技术在高速率、网络化、多用途、 一体化、多谱段 5 个方面的发展趋势。为进一步推动空间激光通信技术研究和产业化的发展, 本文从实施基础研究计划、重 视核心元器件研发、积极参与国际技术标准的制定以及引导相关产业发展 4 个方面提出了发展建议, 以期更好地促进我国空 间激光通信技术的成果转化和应用。
\end{abstract}

关键词：空间激光通信；天地一体化信息网络；高速率；激光组网；一对多激光通信

中图分类号: TN249 文献标识码：A

\section{Progress and Prospect of Space Laser Communication Technology}

\section{Wang Tianshu, Lin Peng, Dong Fang, Liu Xianzhu, Ma Wanzhuo, Fu Qiang}

(National and Local Joint Engineering Research Center of Space Optoelectronics Technology, Changchun University of Science and Technology, Changchun 130022, China)

\begin{abstract}
Space laser communication technology is a major communication technology for space broadband information transmission in the future and has the advantages of high bandwidth, fast and convenient transmission, and low cost. It is the best means to cover the "last kilometer" of information transmission. This study aims to systematically understand the development process of the space laser communication technology. It summarizes the development of research and experimental verification of the technology in China and abroad regarding satellite-ground, inter-satellite, space-ground, and inter-space links. The key technologies of laser communication are studied in detail regarding acquisition tracking, communication transceiving, atmospheric compensation, and optomechanical design. Based on this, five future development trends of space laser communication are summarized emphatically, that is, high speed, networking, multi-purpose, integration, and multi-band. To further promote the research and industrialization of the space laser communication technology, this study proposes implementing basic research plans, focusing on the research and development of core components, actively participating in the formulation of international technical standards, and guiding the development of related industries. Keywords: space laser communication; space-earth integration network; high speed rate; laser networking; one-to-multiple laser communication
\end{abstract}

收稿日期 : 2020-03-12; 修回日期 : 2020-05-11

通讯作者: 王天枢, 长春理工大学空间光电技术国家地方联合工程研究中心教授, 研究方向为空间激光通信、光纤激光技术及应用研究; E-mail: wangts@cust.edu.cn

资助项目：中国工程院咨询项目 “我国激光技术与应用 2035 发展战略研究” (2018-XZ-27); 国家自然科学基金项目 “基于锁模掺钬光纤激 光器的空间高速信息传输特性研究” (61975021)

本刊网址：www.engineering.org.cn/ch/journal/sscae 


\section{一、前言}

空间激光通信技术结合了无线电通信和光纤通 信的优点, 以激光为载波进行通信。空间激光通信 技术具有抗干扰能力强、安全性高、通信速率高、 传输速度快、波段选择方便及信息容量大的优势, 其特点是系统体积小、重量轻、功耗低、施工简单、 灵活机动, 在军事和民用领域均有重大的战略需求 与应用价值 $[1,2]$ 。

空间激光通信技术可作为一种应急通信方案, 应用于抗震救灾、突发事件、反恐、公安侦查等领 域。具体来看, 空间激光通信技术可为多兵种联合 攻防提供军事保密信息服务, 在局部战争、战地组 网和信息对抗中优势突出。另外, 受益于带宽高、 传输快速便捷及成本低的优势, 空间激光通信技术 是解决信息传输 “最后一千米” 和第五代移动通信 技术（5G）小微基站传输的最佳选择 [3]。我国天 地一体化信息网络工程是落实 “没有网络安全, 就 没有国家安全” 的重大建设项目，包括空间网络的 宽带骨干网、接入网等宽带空间信息传输 [4], 但 由于传统微波卫星通信方式很难满足空间网络最高 传输宽带 40 100 Gb/s 的需求, 亟需建设空间激光 网络来支撑这项重大工程。

鉴于空间激光通信技术发展的重要性和紧迫 性, 亟需对此开展全面的深入研究。基于此, 本文 梳理空间激光通信技术的发展现状，分析其关键技 术情况, 研判其未来发展趋势, 并就此提出促进我 国激光通信技术快速发展的对策建议。

\section{二、空间激光通信技术的发展现状}

目前，空间激光通信技术已在多种链路成功开 展了试验, 如卫星 / 地面、卫星 / 卫星、卫星 / 飞机、 飞机/飞机、飞机/地面及地面站间等 [5]。美国、 欧洲、日本、中国和俄罗斯等国家和地区在空间激 光通信关键技术领域均已取得突破, 且进行了多项 试验验证（见图 1), 积极推动空间激光通信技术的 实际应用。

\section{（一）国外主要发展现状}

（1）美国在国家航空航天局（NASA）和空 军支持下是最早开展空间激光通信技术研究的国

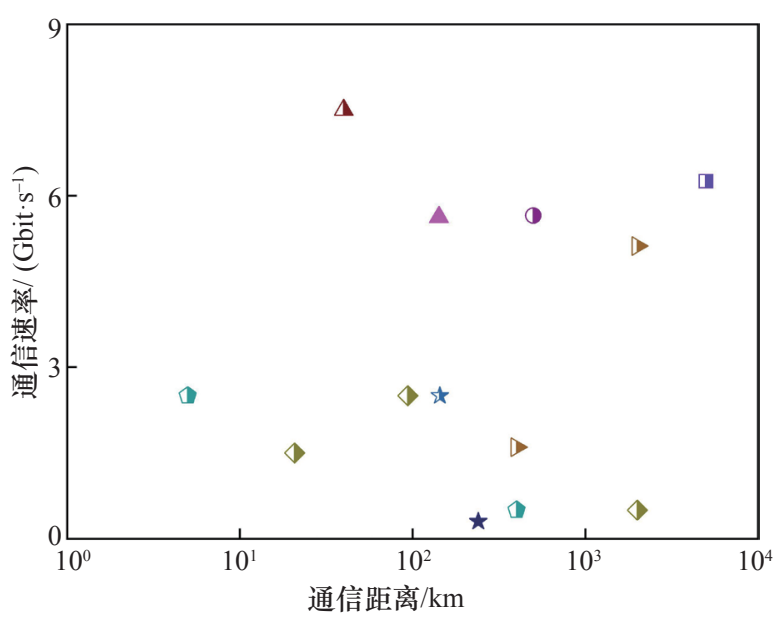

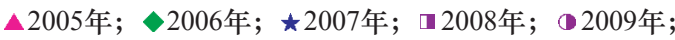

\2010年；2011年； 2013年；2014年； 2016年

图 1 空间激光通信技术试验成果

家。具体来看, 2000 年, NASA 依托喷气推进实验 室完成了激光通信演示系统（OCD）试验；2013 年 10 月的月球激光通信演示验证计划 (LLCD) 实 现了月球轨道与多个地面基站 $4 \times 10^{5} \mathrm{~km}$ 的激光双 向通信 [6,7], 月地最大下行和上行速率分别达到 $622 \mathrm{Mb} / \mathrm{s}$ 和 $20 \mathrm{Mb} / \mathrm{s} ; 2017$ 年 11 月, NASA 创新 型 $1.5 \mathrm{U}$ 立方体卫星的 “激光通信与传感器演示” (OCSD) 项目对未来小型卫星的高速率激光数据传 输技术进行了验证 [8], 星地链路下行速率最高达到 $2.5 \mathrm{~Gb} / \mathrm{s}$ 。

(2) 欧洲的主要国家和地区也较早地开展了 空间激光通信技术的研究。具体有：欧洲航天局 (ESA) 在 2001 年实施半导体激光星间链路试验 (SILEX) 项目, 首次验证了低轨道 (LEO) 卫星至 地球同步轨道（GEO）卫星间的通信; 2008 年，德 国航空航天中心 (DLR) 利用 Tesat 开展了 GEOLEO 远距离空间激光通信在轨原理试验验证, 传输 距离为 $45000 \mathrm{~km}$, 天线口径为 $135 \mathrm{~mm}$, 采用的是 $1.06 \mu \mathrm{m}$ 载波的二进制相移键控（BPSK）相干技 术 [9], 最高速率达 $5.625 \mathrm{~Gb} / \mathrm{s}$, 误码率小于 $10^{-8}$; 2015 年, 德国建立了车载自适应光学通信地面 站, 实现了车载自适应激光通信终端与 LEO 的高 速率传输, 传输速率为 $5.625 \mathrm{~Gb} / \mathrm{s}$, 同时实现了与 地球同步卫星 Alphasat 激光通信终端之间带宽为 $2.8125 \mathrm{~Gb} / \mathrm{s}$ 、有效速率为 $1.8 \mathrm{~Gb} / \mathrm{s}$ 的双向激光通 信 $[10,11]$ 。

（3）日本已经开展了一系列星地激光通信 
演示验证，如工程试验卫星（ETS-VI，19951996 年) 计划和光学在轨测试通信卫星 (OICETS, 2003/2006 年) 计划都完成了激光通信测试, 实现了 世界首次 LEO 卫星与移动光学地面站间的激光传 输 $[12,13]$ 。另外, 日本的相关研究已逐步向激光通 信终端小型化、轻量化、低功耗方向发展, 如通过 空间光通信研究先进技术卫星计划 (SOCRATES), 并在 2014 年完成了小型光学通信终端（SOTA）对 地激光通信在轨测试 [14], SOTA 总质量仅为 $5.8 \mathrm{~kg}$, 最远通信距离达 $1000 \mathrm{~km}$, 下行通信速率为 $10 \mathrm{Mb} / \mathrm{s}$ 。

\section{（二）国内发展现状}

我国在空间激光通信技术领域的研究起步较 晚, 但近年来成果显著, 如在通信系统技术和端机 研制方面取得重大突破, 在激光通信单元技术领域 取得不少研究成果。

我国空间激光通信技术的研究进展如下。 2007 年, 我国首次完成了动中通空间激光通信试 验, 突破了双动态光束瞄准跟踪技术, 传输速率达 $300 \mathrm{Mb} / \mathrm{s}$, 并逐渐将速率提高到 $1.5 \mathrm{~Gb} / \mathrm{s} 、 2.5 \mathrm{~Gb} / \mathrm{s}$ 、 $10 \mathrm{~Gb} / \mathrm{s}$, 陆续开展了空地、空空等链路的演示验 证 [15]; 2013 年完成了两架固定翼飞机间远距离 激光通信试验, 传输速率为 $2.5 \mathrm{~Gb} / \mathrm{s}$, 距离突破 $144 \mathrm{~km}$ ，超过了欧洲、美国等国家和地区同类型演 示验证的最远距离 [16]。2011 年, 通过 “海洋二号” 卫星开展了我国首次星地激光通信链路的数据传输 在轨测试 [17], 最高下行速率为 $504 \mathrm{Mb} / \mathrm{s}$ 。2017 年, 利用 “墨子号” 量子科学实验卫星开展了我国首 次星地高速相干激光通信技术在轨试验 [18], 最高 下行速率达到 $5.12 \mathrm{~Gb} / \mathrm{s}$ 。2017 年, 搭载 “实践 十三号” 高通量卫星的星地激光通信终端开展的我 国首次高轨卫星对地高速激光双向通信试验取得成 功 [19], $40000 \mathrm{~km}$ 星地距离最高速率为 $5 \mathrm{~Gb} / \mathrm{s}$ 。这 些空间通信试验在系统设计、捕获跟踪技术和光波 的大气传输特性等方面为我国空间激光通信技术的 研究提供了宝贵的经验。

\section{三、空间激光通信技术关键技术分析}

随着激光、光学和光电子元器件技术的发展进 步, 空间激光通信技术不断取得突破。按照系统功
能划分，空间激光通信技术主要分为捕获跟踪、通 信收发、大气补偿和光机电设计 4 类技术, 具体介 绍如下。

\section{（一）捕获跟踪技术}

空间激光通信技术借助光源的小发散角波束 提供高功率增益, 这对光束的捕获跟踪提出了比 微波通信更高的要求。实现快速、大概率、大范 围的光束捕获和稳定的高带宽、高精度光束跟踪 是空间激光通信瞄准、捕获、跟踪技术研究的核 心目标。其中, 光束捕获采用激光瞄准技术和粗 / 精跟踪相独立的体制, 即粗跟踪由大视场相机和 伺服转台组成闭环，提供大范围低频带伺服控制; 精跟踪由高帧频相机和快速振镜组成闭环，提供 小范围高频带伺服控制，从而有效抑制因光束大 范围运动和高频率抖动引起的光束扰动。

随着激光技术的进步, 受益于激光光束智能 变换、激光相控阵等新技术的逐渐发展成熟, 将 其应用于空间激光通信技术的捕获、瞄准、跟踪 系统中，使传统跟瞄模式发生改变，可提高空间 光通信系统的跟瞄精度、速度和可靠性。同时, 小型高效率激光器的出现也使跟瞄系统向小型化、 轻型化和集成化发展。另外, 可采用粗精复合高 精度跟踪, 通过激光光束智能变换, 在保证跟踪 性能的前提下，简化激光通信跟瞄系统。

\section{（二）通信收发技术}

空间激光通信技术需要激光器具有大调制带 宽、高发射功率和窄线宽等特点。具体来看, 激光 调制技术的调制方式可以分为直接调制和间接调 制, 由于直接调制方式使带宽和发射功率受限, 目 前主要采用小功率种子激光源间接调制后通过高功 率光纤放大器获得高发射功率的方法进行调制; 根 据作用光束的参数不同 (如强度、频率、相位等), 可分为调幅、调频和调相等不同调制方式，由于 不同波长系统相应器件的差异, 调制方式也有所差 别。目前空间激光通信技术采用的激光波长主要 有 $800 \mathrm{~nm} 、 1000 \mathrm{~nm}$ 和 $1550 \mathrm{~nm} 3$ 个波段, 其中 $800 \mathrm{~nm}$ 波段的半导体激光器一般利用强度调制/ 直接检测 (IM/DD)，1000 nm 波段的 Nd:YAG 固 体激光器可采用各种调制方式, 而 $1550 \mathrm{~nm}$ 波段 
的半导体激光器与光纤通信系统兼容, 可采用多 种高速调制方式并利用掺铒光纤放大器实现高速、 高功率发射。

激光通信接收机的高速探测器均由光纤耦合以 适应高速探测器的小探测截面, 并有利于系统集成 化。因此, 空间激光到光纤的耦合是激光通信接收 部分的关键技术之一，其中对光纤高效率耦合主要 受模式匹配、对准偏差、菲涅尔反射、吸收损耗、 平台振动等影响。现有的光纤耦合方法主要采用的 是光学自适应、雉形光纤、光纤章动等, 尚未出现 实质性突破，光纤高效耦合技术仍是当前空间激光 通信系统的主要难题之一。

\section{（三）大气补偿技术}

当空间激光通信技术应用在星地、空空和空地 等链路时, 激光在穿越大气层的过程中受大气湍流 影响, 在传输时会出现接收功率抖动, 导致系统出 现误码, 这在高速激光通信中更加明显 [20]。为解 决这一问题, 采用高精度实时波前畸变校正技术是 抑制大气湍流对传输光束波前影响的有效方法, 即 通过哈特曼传感器进行多孔径波面探测, 在一定程 度上能够矫正波前畸变。但该技术的主要难点在于 激光到达角起伏补偿、波面变形补偿和空中飞行时 附面层影响补偿, 可通过探测系统引入波前畸变补 偿镜技术进行联合校正。

近年来有关大气信道的研究成果颇丰。例如, 2018 年开展的基于部分相干载波的大气高速传输 研究, 由有源锁模光纤激光器百浦色散位移光纤而 产生的超连续谱光源作为部分相干高速载波 [21], 在 $1 \mathrm{~km}$ 大气湍流信道中, 相比于相干光源, 采用 部分相干光载波源能有效抑制大气湍流造成的光强 闪炼。而后，证明了可将全光时分复用 (OTDM) 技术应用在部分相干光通信系统中以提升传输速 率 [22], 最高速率达到了 $16 \mathrm{~Gb} / \mathrm{s}$ 。

\section{（四）光机电设计技术}

为减小自由空间的功率损耗, 提高发射光学系 统增益, 需要通信光束以近衍射极限角发射。在保 证发射光学口径的基础上, 提高光束发射增益对光 纤耦合技术、光束整形技术、望远镜面型设计提出 了更严格的要求。为突破近衍射极限角发射的关键 技术、发射激光源的整形准直技术和高效率光纤耦
合技术，亟需通过激光技术的发展，研究光纤不同 芯径、束散角与光学系统匹配的优化选取方法。

对于光学基台技术, 要求对光学系统进行模块 化、轻量化设计, 且能满足未来空间激光通信网络 一点对多点动中通同时传输 [23]。与此同时, 激光 技术的广泛应用促成了多行业的标准化，如基于激 光技术的激光整形传输促成了元件的模块化和标准 化，降低了整机体积与成本。

\section{四、空间激光通信技术的发展趋势}

\section{(一) 高速率}

随着空间激光通信高速调制解调和传输技术 的快速发展, 未来星地激光通信链路速率有望达 到 $100 \mathrm{~Gb} / \mathrm{s}$ 量级。高速激光通信采用高阶调制方式 如正交相移键控（QPSK）、正交振幅调制（QAM） 和复用方式如波分复用 (WDM)、时分复用 (TDM)、 轨道角动量复用 $(\mathrm{OAM})$, 短距离 $(<1 \mathrm{~km})$ 速率 可达 $\mathrm{Tb} / \mathrm{s}$ 量级。近年来, 国内外高速无线激光通信 技术的主要发展现状如图 2 所示。

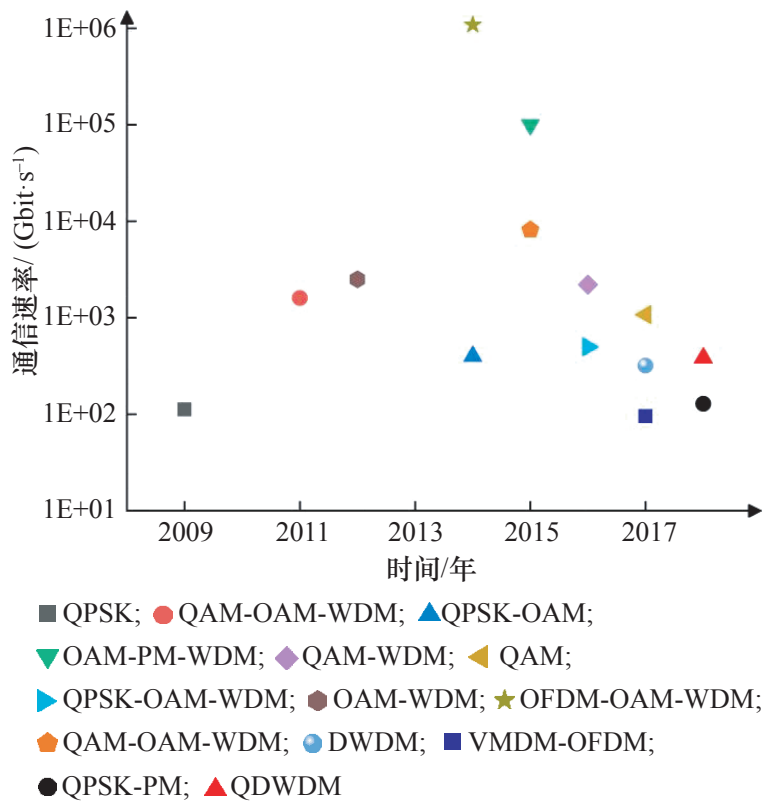

图 2 国内外高速无线激光通信技术的主要发展现状 注：1E+0n 表示 $1 \times 10^{\mathrm{n}}$; QPSK 代表正交相移键控; QAM-OAM-WDM 代表 正交振幅调制 - 轨道角动量复用 - 波分复用; QPSK-OAM 代表正交相移键控 轨道角动量复用: OAM-PM-WDM 代表轨道角动量复用 - 偏振复用 - 波分复 用; QAM-WDM 代表正交振幅调制 - 波分复用; QAM 代表正交振幅调制; QPSK-OAM-WDM 代表正交相移键控 - 轨道角动量复用 - 波分复用; OAMWDM 代表轨道角动量复用 - 波分复用; OFDM-OAM-WDM 代表正交频分 复用 - 轨道角动量复用 - 波分复用; QAM-OAM-WDM 代表正交振幅调制 轨道角动量复用 - 波分复用; DWDM 代表密集波分复用; VMDM-OFDM 代 表矢量模分复用 - 正交频分复用; QPSK-WDM 代表正交相移键控 - 波分复 用; QPSK-PM 代表正交相移键控; QDWDM 代表密集波分复用。 
国外在空间激光通信技术高速率方面的主要研 究进展有: 2009 年, 日本电气株式会社（NEC）实 验室利用 QPSK 和多路输入 / 输出 (MI/MO) 相 干检测方法实现了 $112 \mathrm{~Gb} / \mathrm{s}$ 试验 [24]; 南加州大 学采用 12 路 QAM-2 路偏振 -42 路波分技术在室 内 $1 \mathrm{~m}$ 的距离上实现了 $100 \mathrm{~Tb} / \mathrm{s}$ 自由空间光通 信 [25]。2016 年, 沙特国王大学采用 12 路 WDM 和 16-QAM 调制技术在室外 $11.5 \mathrm{~m}$ 的距离上进行 了 $2.2 \mathrm{~Tb} / \mathrm{s}$ 自由空间光高速通信实验 [26], 并在 2017 年采用 $3.6 \mathrm{~b} / \mathrm{s} / \mathrm{Hz}$ 的频谱效率 32-QAM 调制在室 外干燥的沙漠地区 $100 \mathrm{~m}$ 的距离上突破了 $1.08 \mathrm{~Tb} / \mathrm{s}$ 自由空间光通信 [27]。

通过国际合作交流, 我国在空间激光通信技 术高速率方面的研究取得了重要进展 (见图 2)。 具体有: 华中科技大学开展了一系列 OAM 超高 速无线光传输试验, 实验室内最高传输速率可达 $1.086 \mathrm{~Pb} / \mathrm{s}$ [28 30]。该研究是在较短距离上的超高 速无线光传输, 但传输速率方面已达到了国际领先 水平。另外, 在长距离高速空间激光传输研究方面 也取得了一定进展, 如 2018 年长春理工大学与浙 江大学合作, 采用 3 路密集波分复用 (DWDM) 的 QPSK 调试单路载波 $40 \mathrm{~Gb} / \mathrm{s}$, 在 $1 \mathrm{~km}$ 距离开展了 速率 $120 \mathrm{~Gb} / \mathrm{s}$ 的自由空间光通信实验 [31], 而后 又突破了单路载波 $128 \mathrm{~Gb} / \mathrm{s}$ 和 3 路 DWDM 总速率 $384 \mathrm{~Gb} / \mathrm{s}$ 大气传输 $[32,33]$ 。

\section{(二) 网络化}

随着全球化和信息技术的发展，亟需建设具有 不依托地面网络、无缝覆盖全球、高带宽和抗毁性 能的空间网络。因此, 依托空间激光通信技术实现 的天基宽带传送网络是今后发展的重要趋势。

空间激光通信技术逐渐从点对点模式向中继转 发和构建激光网络的方向发展。由于激光网络建设 的主要难点在于激光发散角小、光信号动态接入以 及受空间环境影响大等, 因此构建激光通信网络时, 需突破 “一对多” 的激光通信技术难题、研究动态 路由解决接入方案、寻求激光通信和微波联合通信 体制。长春理工大学提出的采用旋转抛物面结构设 计一点对多点光学收发天线, 实现多颗卫星间激光 通信组网, 光学原理简单, 是探索解决这一难点的 重大突破 [34]。

\section{（三）多用途}

随着空间激光通信技术的逐渐成熟，空间激光 通信的高调制速率、远传输距离和低能耗的优点逐 渐凸显。目前, 空间激光通信技术已广泛应用于星 间、星空、空空、空地等链路的宽带数据传输, 并 逐渐向深空探测、水下和地面接入通信扩展, 用途 越来越广。

深空探测是人类对月球、远距离天体或空间开 展的探测活动, 是了解太阳系及宇宙, 揭示宇宙起 源与演变, 拓展人类生存空间的必然选择。月球探 测工程的实施拉开了我国深空探测的序幕, 随后又 实施了火星探测工程 $[35,36]$ 。水下无线光通信作为 一种新兴通信技术, 具有容量大、带宽高、保密性 好、抗干扰能力强等优势, 已成为世界大国竞相发 展的一项重要通信技术 [37]。利用可见光进行数据 通信的无线光传输技术兼具照明、通信和控制定位 等功能, 易与现有基础照明设施相融合, 且符合国 家节能减排的战略思想, 逐渐成为未来智能时代超 高速泛在光联网的主要宽带传输方法。另外, 在一 些无法铺设光缆的特殊应用场合, 如海岛之间、城 市楼宇间、野外复杂环境等, 空间激光通信技术可 起到光纤通信技术所无法替代的作用。

\section{（四）一体化}

由于激光在高速通信和精密测距方面具有优 势, 近年来激光测距与通信一体化技术越来越受到 重视。激光测距与通信一体化设计是以高速通信为 主, 兼顾精密测距, 使用同一束激光和硬件平台实 现测距和信息传输, 进而实现同一套设备完成测距 和通信的双重功能。2013 年 NASA 的 LLCD 系统 已经成功实施月地高速激光通信与高精度测距的在 轨演示验证 [38], 测距精度达到 $3 \mathrm{~cm} ; 2014$ 年, 北 京遥测技术研究所完成了基于相干通信的测距和高 速通信一体化的设计 [39]; 2015 年, 长春理工大学 提出了空间目标测距、成像、通信一体化方案 [40], 其中激光通信信标光发射/接收和激光测距光发射 / 接收共用一个光学天线。

此外, 激光和微波通信技术的融合, 也是目前 学术研究的热点, 主要包括激光与微波收发融合、 数据处理融合、微波信号的激光调制和产生等。目 前, 微波光子技术逐渐发展成熟, 并已应用于雷达 
信号的激光传输和处理, 未来该技术也将在激光与 微波融合通信系统中应用。

\section{（五）多谱段}

随着多种光谱段激光技术的进步, 从紫外到红 外, 甚至是太赫兹波段, 均已出现了可应用的激光 技术。由于各谱段在抗电磁干扰、云雾穿透能力、 自组网等方面的优势各有不同, 因此, 未来空间激 光通信利用不同谱段通信系统的优势, 将大力发展 紫外、可见、中红外、太赫兹等多谱段结合的通信 模式。同时, 加强多谱段通信的深入研究, 以期通 过多谱段联合应用来实现不同环境条件下的不间断 无障碍通信。

与空间激光通信技术相比, 紫外无线光通信技 术无需严格的捕获、瞄准、跟踪就可实现非直视通 信, 在自组网、复杂电磁环境及特殊地形等应用中 优势明显。加州理工大学于 2009 年理论研究了紫 外光散射模型及探测器、调制方式的影响并进行了 试验验证 [41]。太赫兹无线光通信方面, 近年来也 取得了突破性成果, 中国工程物理研究院的太赫兹 科研团队于 2017 年利用频率 $0.14 \mathrm{THz}$ 载波, 成功 开展了单路实时速率 $5 \mathrm{~Gb} / \mathrm{s}$ 的 $21 \mathrm{~km}$ 远距离高速无 线传输试验 [42]。

\section{五、政策建议}

目前, 国内外在空间激光通信技术领域的发展 均未形成规模，鉴于该技术的巨大发展潜力，世界 各国积极投入人力、物力开展相关研究, 并引导相 关产业发展。近年来, 欧洲、美国等国家或地区已 在空间激光通信技术方面对星地、星间、空空等链 路进行了部分商用化测试, 我国也完成了相关的演 示验证工作。因此，在这一领域我国与欧美等发达 国家和地区的差距较小，在某些方面还具有一定的 后发优势, 但核心元器件依赖进口的问题尚未解决。 为此, 通过国家政策倾斜, 加强顶层设计, 重点突 破卡脖子技术难题, 推进空间激光通信技术产业化, 使我国在该领域能够追赶甚至是达到领先水平。为 推动空间激光通信技术的发展, 提出如下政策建议。

\section{（一）实施基础研究计划}

空间激光通信技术的发展沿用了很多光纤通信
及光学设计的技术，但根据新的应用特点会形成新 的颠覆性技术, 需要加强基础研究以取得突破。因 此, 建议尽快实施以高等院校基础科研为主的无线 激光宽带传输与组网基础科学问题研究计划, 在重 点核心技术上取得突破, 使我国尽快在该领域的基 础研究和关键技术达到世界领先水平。

\section{（二）重视核心元器件的研发}

光电子、光学核心元器件的工艺水平制约了我 国空间激光通信技术的发展, 且进口依赖度高。因 此, 建议组织元器件研究单位、高等院校及企业开 展关键技术攻关，并大力扶持技术成果转化。利用 我国在光纤通信技术和产业领域引领世界技术与产 品创新优势, 面向未来空间信息网络应用, 努力实 现核心元器件的自主知识产权。

\section{（三）积极参与空间激光通信技术标准的制定}

随着空间激光通信技术的成熟并逐渐商用化, 其技术标准的制定尤为重要。因此, 建议从国家层 面积极引导实施空间激光通信技术标准计划。通过 组织高等院校、科研机构及相关企业开展技术标准 研究, 积极参与国际标准的制定, 促进我国空间激 光通信技术和产业化的发展。

\section{（四）引导相关产业的形成和发展}

随着空间激光通信技术的不断进步和应用领域 的不断扩展, 相关产业已逐渐形成, 需正确地引导 并促进产业健康发展。因此, 建议对我国空间激光 通信技术领域的产业发展进行合理规划, 引导高等 院校、科研机构和企业开展产业合作，利用基础研 究和关键技术优势形成有效的成果转化, 并促进产 业快速发展壮大。

\section{参考文献}

[1] 姜会林, 安岩, 张雅琳, 等. 空间激光通信现状、发展趋势及关键 技术分析 [J]. 飞行器测控学报, 2015, 34(3): 207-217.

Jiang H L, An Y, Zhang Y L, et al. Analysis of the status quo, development trend and key technologies of space laser communication [J]. Journal of spacecraft TT \& C Technology, 2015, 34(3): 207-217.

[2] 高铎瑞, 李天伦, 孙悦, 等. 空间激光通信最新进展与发展趋势 [J]. 中国光学. 2018, 11(6): 901-913.

Gao D R, Li T L, Sun Y, et al. Latest developments and trends of space laser communication [J]. Chinese Optics, 2018, 11(6): 901-913. 
[3] 杨乾远, 孙晖, 马拥华, 等. $5 \mathrm{G}$ 基站前传和中传的无线光通信方 案设计 [J]. 光通信技术, 2019, 43(9): 23-26.

Yang Q Y, Sun H, Ma Y H, et al. Design of free space optical communication scheme for forward and intermediate transmission of $5 \mathrm{G}$ base station $[\mathrm{J}]$. Optical Communication Technology, 2019, 43(9): 23-26.

[4] 姜会林, 江伦, 宋延嵩, 等. 一点对多点同时空间激光通信光学 跟瞄技术研究 [J]. 中国激光, 2015, 42(4): 1-9.

Jiang H L, Jiang L, Song Y S, et al. Research of optical and apt technology in one-point to multi-point simultaneous space laser communication system [J]. Chinese Journal of Lasers, 2015, 42(4): $1-9$.

[5] 姜会林, 付强, 赵义武, 等. 空间信息网络与激光通信发展现状 及趋势 [J]. 物联网学报, 2019, 3(2): 1-8.

Jiang H L, Fu Q, Zhao Y W, et al. Development status and trend of space information network and laser communication [J]. Chinese Journal on Internet of Things, 2019, 3(2): 1-8.

[6] Grein M E, Kerman A J, Dauler E A, et al. Design of a groundbased optical receiver for the lunar laser communications demonstration [C]. Santa Monica: 2011 International Conference on Space Optical Systems and Applications (ICSOS), 2011.

[7] Boroson D M, Robinson B S, Burianek D A, et al. Overview and status of the lunar laser communications demonstration [C]. Society of Photo-Optical Instrumentation Engineers, 2012.

[8] The Aerospace Corporation of El Segundo, California. Update on Optical communications and sensor demonstration (OCSD) [EB/ OL]. (2017-11-02)[2020-05-06]. https://www.nasa.gov/feature/ ocsd.

[9] Fields R, Kozlowski D, Yura H, et al. 5.625 Gbps bidirectional laser communications measurements between the NFIRE satellite and an optical ground station [C]. Santa Monica: 2011 International Conference on Space Optical Systems and Applications (ICSOS), 2011.

[10] Seel S, Kämpfner H, Heine F, et al. Space to ground bidirectional optical communication link at 5.6 Gbps and EDRS connectivity outlook [C]. Big Sky: 2011 Aerospace Conference, 2011.

[11] Tröndle D, Pimentel P M, Rochow C, et al. Alphasat-Sentinel-1A optical inter-satellite links: Run-up for the European data relay satellite system [C]. Society of Photo-Optical Instrumentation Engineers, 2016.

[12] Arimoto Y, Toyoshima M, Toyoda M, et al. Preliminary result on laser communication experiment using Engineering Test SatelliteVI (ETS-VI) [C]. San Jose: Free-Space Laser Communication Technologies VII, 1995.

[13] Jono T, Takayama Y, Ohinata K, et al. Demonstrations of ARTEMIS-OICETS inter-satellite laser communications [C]. San Diego: Aiaa International Communications Satellite Systems Conference, 2006.

[14] Carrasco-Casado A, Takenaka H, Kolev D, et al. LEO-toground optical communications using SOTA (Small Optical TrAnsponder) - Payload verification results and experiments on space quantum communications [J]. Acta Astronautica, 2017, 139: 377-384

[15] 吴从均, 颜昌翔, 高志良, 等. 空间激光通信发展概述 [J]. 中国 光学, 2013, 6(5): 670-680.

Wu C J, Yan C X, Gao Z L, et al. Overview of space laser com- munications [J]. Chinese Optics, 2013, 6(5): 670-680.

[16] 王岭, 陈䂀, 董峰. 空间激光通信光端机发展水平与发展趋势 [J]. 长春理工大学学报(自然科学版), 2016, 39(2): 39-45.

Wang L, Chen X, Dong F. Development level and trend for space laser communication optical transceiver [J]. Journal of Changchun University of Science and Technology (Natural Science Edition), 2016, 39(2): 39-45.

[17] 吴应明, 刘兴, 罗广军, 等. 空间光通信网络技术的研究进展及 架构体系 [J]. 光通信技术, 2017, 11(12): 46-49.

Wu Y M, Liu X, Luo G J, et al. Research progress and structure system of space optical communication network technology [J]. Optical Communication Technology, 2017, 11(12): 46-49.

[18] 任建迎, 孙华燕, 张来线, 等. 空间激光通信发展现状及组网新 方法 [J]. 激光与红外, 2019, 49(2): 143-150.

Ren J Y, Sun H Y, Zhang L X, et al. Development status of space laser communication and new method of networking [J]. Laser and Infrared, 2019, 49(2): 143-150.

[19] 王旭. 实践十三号卫星成功发射开启中国通信卫星高通量时代 [J]. 中国航天, 2017 (5): 13.

Wang X. The successful launch of Shijian-13 satellite opens the high throughput era of China's communication satellite [J]. Aerospace China, 2017 (5): 13.

[20] 陈纯毅, 杨华民, 姜会林, 等. 大气光通信中大孔径接收性能分 析与孔径尺寸选择 [J]. 中国激光, 2009, 36(11): 2957-2961.

Chen C Y, Yang H M, Jiang H L, et al. Performance analysis of large-aperture receiving and selection of aperture size in atmospheric optical communications [J]. Chinese Journal of Lasers, 2009, 36(11): 2957-2961.

[21] Zhang X M, Wang T S, Chen J D, et al. Scintillation index reducing based on wide-spectral mode-locking fiber laser carriers in a simulated atmospheric turbulent channel [J]. Optics Letters, 2018, 43 (14): 3421-3424.

[22] Chen J D, Wang T S, Zhang X M, et al. Free-space transmission system in a tunable simulated atmospheric turbulence channel using a high-repetition-rate broadband fiber laser [J]. Applied Optics, 2019, 58 (10): 2635-2640.

[23] 付强, 姜会林, 王晓曼, 等. 空间激光通信研究现状及发展趋势 [J]. 中国光学, 2012, 5(2): 116-125.

Fu Q, Jiang H L, Wang X M, et al. Research status and development trend of space laser communication [J]. Chinese Optics, 2012, 5(2): 116-125.

[24] Cvijetic N, Qian D Y, Yu J J, et al. $100 \mathrm{~Gb} / \mathrm{s}$ per-channel freespace optical transmission with coherent detection and MIMO processing [C]. Vienna: 2009 35th European Conference on Optical Communication, 2009.

[25] Huang H, Xie G D, Yan Y, et al. $100 \mathrm{~Tb} / \mathrm{s}$ free-space data link enabled by three-dimensional multiplexing of orbital angular momentum, polarization, and wavelength [J]. Optics Letters, 2014, 39(2): 197-200.

[26] Esmail M A, Ragheb A, Fathallah H, et al. Experimental demonstration of outdoor 2.2 Tbps super-channel FSO transmission system [C]. Kuala Lumpur: 2016 IEEE International Conference on Communications Workshops (ICC), 2016.

[27] Esmail M A, Ragheb A, Fathallah H, et al. Investigation and demonstration of high speed full-optical hybrid FSO/fiber communication system under light sand storm condition [J]. IEEE 
Photonics Journal, 2016, 9(1): 1-12.

[28] Wang J, Yang J Y, Fazal I M, et al. Terabit free-space data transmission employing orbital angular momentum multiplexing [J]. Nature photonics, 2012, 6(7): 488-496.

[29] Wang J, Li S, Luo M, et al. N-dimentional multiplexing link with $1.036-\mathrm{Pb} / \mathrm{s}$ transmission capacity and $112.6-\mathrm{b} / \mathrm{s} / \mathrm{Hz}$ spectral efficiency using OFDM-8QAM signals over 368 WDM polmuxed 26 OAM modes [C]. Cannes: 2014 European Conference on Optical Communication (ECOC), 2014.

[30] Wang J, Liu J, Lv X, et al. Ultra-high 435-b/s/Hz spectral efficiency using $\mathrm{N}$-dimentional multiplexing and modulation link with pol-muxed 52 orbital angular momentum (OAM) modes carrying Nyquist 32-QAM signals [C]. Valencia: 2015 European Conference on Optical Communication (ECOC), 2015.

[31] Gao S M, Feng S L, Wu Z H, et al. 120 Gb/s high-speed WDMQPSK free-space optical transmission through a 1-km atmospheric channel [J]. Electronics Letters, 2018, 54 (18): 1082-1084.

[32] Liu X, Wang T, Zhang X, et al. $128 \mathrm{~Gb} / \mathrm{s}$ free-space laser transmission performance in a simulated atmosphere channel with adjusted turbulence [J]. IEEE Photonics Journal, 2018, 10(2): 1-10.

[33] Liu X, Wang T, Lin P, et al. Up to $384 \mathrm{Gbit} / \mathrm{s}$ based on dense wavelength division multiplexing of $100-\mathrm{GHz}$ channel spacing free space laser transmission performance in a simulated atmosphere channel with adjusted turbulence [J]. Optical Engineering, 2018, 57(10): 1-6.

[34] 姜会林, 胡源, 丁芗, 等. 空间激光通信组网光学原理研究 [J]. 光 学学报, 2012, 32(10): 1-5.

Jiang H L, Hu Y, Ding Y, et al. Optical principle research of space laser communication network [J]. Acta Optica Sinica, 2012, 32(10): $1-5$.

[35] 吴伟仁, 于登云. 深空探测发展与未来关键技术 [J]. 深空探测 学报, 2014, 1(1): 5-17.

Wu W R, Yu D Y. Development of deep space exploration and its future key technologies [J]. Journal of Deep Space Exploration,
2014, 1(1): 5-17.

[36] 于登云, 吴学英, 吴伟仁. 我国探月工程技术发展综述 [J]. 深空 探测学报, 2016, 3(4): 307-314.

Yu D Y, Wu X Y, Wu W R. Review of technology development for Chinese lunar exploration program [J]. Journal of Deep Space Exploration, 2016, 3(4): 307-314.

[37] Khalighi M A, Gabriel C, Hamza T, et al. Underwater wireless optical communication: Recent advances and remaining challenges [C]. Graz: 2014 16th International Conference on Transparent Optical Networks (ICTON), 2014.

[38] Boroson D M, Robinson B S, Murphy D V, et al. Overview and results of the lunar laser communication demonstration [C]. Washington: Society of Photo-Optical Instrumentation Engineers, 2014

[39] 刘向南, 李英飞, 向程勇, 等. 激光测距通信一体化技术研究及 深空应用探索 [J]. 深空探测学报, 2018, 5(2): 147-153, 167.

Liu X N, Li Y F, Xiang C Y, et al. Study on integrated technique of laser ranging and communication and its applications in deep space [J]. Journal of Deep Space Exploration, 2018, 5(2): 147153,167

[40] 姜会林, 张国玉, 付强, 等. 空间光电技术与光学系统 [M]. 北京: 科学出版社, 2015.

Jiang H L, Zhang G Y, Fu Q, et al. Space photoelectric technology and optical system [M]. Beijing: China Science Publishing \& Media Ltd., 2015.

[41] Ding H, Chen G, Majumdar A K, et al. Modeling of non-line-ofsight ultraviolet scattering channels for communication [J]. IEEE Journal on Selected Areas in Communications, 2009, 27(9): 15351544.

[42] 吴秋宇, 林长星, 陆涁, 等. $21 \mathrm{~km}, 5 \mathrm{Gbps}, 0.14 \mathrm{THz}$ 无线通信系 统设计与试验 [J]. 强激光与粒子束, 2017, 29(6): 1-4.

Wu Q Y, Lin C X, Lu B, et al. Design and tests of $21 \mathrm{~km}, 5 \mathrm{Gbps}$, $0.14 \mathrm{THz}$ wireless communication system [J]. High Power Laser and Particle Beams, 2017, 29(6): 1-4. 